\title{
Biología reproductiva de Graus nigra (Perciformes, Kyphosidae) en las costas del norte de Chile
}

\author{
Reproductive biology of Graus nigra (Perciformes, Kyphosidae) on the coast of the \\ Northern of Chile
}

Héctor Flores $^{1}$ y Andrés Smith ${ }^{2}$

\begin{abstract}
${ }^{1}$ Departamento de Acuicultura, Facultad de Ciencias del Mar, Universidad Católica del Norte, Larrondo 1281, Coquimbo, Chile. hflores@ucn.cl

${ }^{2}$ Departamento de Biología Marina, Facultad de Ciencias del Mar, Universidad Católica del Norte, Larrondo 1281, Coquimbo, Chile

Abstract: We describe gonad development and spawning time and size at first sexual maturity of Graus nigra, at Northern Chile, using fish collected by spearing from J uly 1998 to J uly 1999. We used macroscopic characterization and histological sections to describe states of gonadic development and we calculated time of spawning, gonadosomatic index (IGS), gonad index (IG) and condition index (K). We estimated size at first maturity using relative increment of IGS and size at $50 \%$ maturity. We found that spawning occurs in spring and size of maturity for females was estimated to be between 39 and $41 \mathrm{~cm}\left(\mathrm{~L}_{\mathrm{t}}\right)$ and for males between 42 and $49 \mathrm{~cm}\left(\mathrm{~L}_{\mathrm{t}}\right)$, depending on the method of analyzis used.
\end{abstract}

Key words: Reproduction, spawning, oocyte, rocky shore fishes

Resumen: Se describe el desarrollo gonadal y se determina la época de desove y la talla de primera madurez reproductiva de Graus nigra en el norte de Chile. Las muestras analizadas fueron recolectadas por buceo apnea con arpón de elásticos en el periodo de julio de 1998 a julio de 1999. La descripción de los estados de desarrollo gonadal se efectuó a través de cortes histológicos y caracterización macroscópica; para la época de desove se determinó el Índice gonadosomático (IGS), gonádico (IG) y de condición (K), para la talla de madurez se aplicó el criterio del incremento relativo del IGS y el método del $50 \%$ de las hembras maduras. La época de desove ocurre en primavera, la talla de madurez para hembras se estimó entre 39 y $41 \mathrm{~cm}\left(\mathrm{~L}_{\mathrm{t}}\right)$ y para machos entre 42 y $49 \mathrm{~cm}$ de $\left(\mathrm{L}_{\mathrm{t}}\right)$, dependiendo del método de análisis.

Palabras clave: Reproducción, desove, oocito, peces litoral rocoso

\section{INTRODUCCIÓN}

La mayoría de las especies de peces que tienen una significativa importancia económica, sea por el volumen de extracción o por su valor comercial, han sido estudiadas desde varios aspectos, donde uno de los más relevantes es la reproducción, antecedentes que permite generar información clave para el manejo y la administración de las pesquerías que ellos sustentan (Balbontin \& Fischer 1981, Pardo \& Oliva 1992, Alarcón \& Arancibia 1993, Arancibia et al. 1994, Claramunt et al. 1994).

La mayoría de los peces desovan en determinados períodos o definidas épocas durante el año, donde la temperatura modula el proceso de desove e incide directamente en la supervivencia de los huevos, larvas y juveniles, existiendo correlación entre esta variable y el desarrollo gonadal (Valdebenito et al. 1995). La mayoría de los peces que habitan zonas templadas, desovan en primavera o principios del verano cuando la temperatura del agua aumenta, como ocurre en Merluccius gayi gayi y Paralichthys microps (Balbontín \& Fischer 1981, Chong
\& González 1995). En otras especies como Sardinops sagax y Polyprion oxygeneios, tienen picos de desove en otoño o invierno, casi siempre coincidente cuando la temperatura es más baja (Flores \& Rojas 1984, Herrera et al.1994). Sin embargo, las especies con una amplia distribución latitudinal pueden presentar diferencias en la época de desove, dependiendo de la localidad y del ambiente en que viven los organismos, como ocurre en Solea solea (Ramos 1982), mientras que especies de bajas latitudes como Lutjanus guttatus, la mayor parte del año se encuentran en estado reproductivo (Arellano-Martínez et al. 2001).

En Chile, los estudios reproductivos en peces se han efectuado preferentemente en las especies de interés comercial y que son extraídas abundantemente, como son Strangomera bentincki, Sardinops sagax, Scomber japonicus peruanus, Merluccius gayi gayi, Trachurus murphyi; los tópicos más tratados han sido el ciclo sexual, época de desove, talla de primera madurez sexual y fecundidad (Balbontín \& Fischer 1981, Pardo \& Oliva 1992, 
Alarcón \& Arancibia 1993, Arancibia et al. 1994, Claramunt et al. 1994, Oyarzún et al. 1998, Vargas \& Castro 2001), aspectos claves para el manejo sustentable de los stocks, permiten generar información que determinan períodos de veda biológica y definen tallas mínimas de extracción, que pueden establecer cuotas de captura, determinando el fragmento de la población susceptible a ser extraída (Sparre \& Venema 1995). También se han estudiado los aspectos reproductivos en especies cuyo nivel de extracción es bajo, pero con un alto valor comercial, tales como Paralichthys microps, P. adspersus, Genypterus blacodes, Xiphias gladius (Angeles 2005, Chong \& González 1995, Zárate 1997, Cordo et al. 2002).

En el caso de las especies ícticas que habitan el litoral rocoso de Chile, los estudios se han centrado principalmente en aspectos relacionados con distribución geográfica, relaciones tróficas, taxonomía y morfometría. Aspectos como dinámica poblacional, determinación de tallas mínimas de extracción o períodos de veda biológica, son antecedentes desconocidos y que constituyen una herramienta fundamental para el buen manejo de los recursos.

Entre los peces del litoral rocoso del norte de Chile se encuentra la vieja negra Graus nigra (Philippi, 1887), especie reconocida primariamente en la familia Labridae (Mann 1954; Moreno \& Castilla 1980), la cual posteriormente en base a estudios de la osteología del aparato bucal, fue incluida en la familia Kyphosidae (Johnson \& Fritzche 1989, Pequeño 1989, Vial \& Ojeda 1992). G. nigra se distribuye latitudinalmente desde el sur del Perú hasta Valdivia en Chile (Vargas \& Pequeño 2004), batimétricamente se encuentra desde el nivel del mar hasta $25 \mathrm{~m}$ de profundidad, siempre en fondos rocosos con grietas y grandes cuevas (Mann 1954, Moreno \& Castilla 1980). $\mathrm{Su}$ cuerpo es generalmente alargado, de tonalidad negruzca resaltando manchas blancas en línea (1 a 4) sobre ambos costados y en la parte posterior del cuerpo, es de cabeza grande, donde la boca se caracteriza por poseer labios gruesos. Posee aleta dorsal única estructurada con espinas en su parte anterior, que continúa con radios blandos. La dentición consta de dientes caniniformes y de placas dentarias, el tubo digestivo es simple, desprovisto de ciegos. Tanto los dientes como el tubo digestivo indican una alimentación carnívora, depredando principalmente sobre equinodermos, crustáceos, moluscos y peces (Moreno 1972, Fuentes 1982, Vargas et al. 1999). Los juveniles habitan pozas intermareales, donde constituyen uno de los componentes más importantes en cuanto a su función y densidad (Varas \& Ojeda 1990, Stepien 1990, Berrios \&
Vargas 2000; Hernández et al. 2002). Durante eventos El Niño muestra un carácter migratorio (Soto-Mamani 1985), lo que podría indicar la preferencia por aguas más bien frías. Es un recurso importante para los buzos mariscadores (Schneider 2008) y deportistas submarinos; las estadísticas muestran variabilidad en las capturas, con 24 ton en promedio anual entre 1995 y 2008 (SERNAPESCA 2010).

Graus nigra se encuentra con síntomas de sobreexplotación (Godoy 2008), y es necesario contar con información biológica para reunir antecedentes y aplicar regulaciones pesqueras y/o investigación de su cultivo. Por lo tanto en este contexto, el objetivo del presente trabajo fue determinar la época de desove y la talla de primera madurez reproductiva en G. nigra, presente en el litoral rocoso del norte de Chile.

\section{MATERIAL Y MÉTODOS}

Se recolectó un total de 251 ejemplares de Graus nigra por medio de buceo apnea con arpón de elásticos. Las capturas se efectuaron desde julio de 1998 a julio de 1999, entre las regiones de Atacama y Coquimbo, desde bahía Sarco (28 $81^{\prime}$ 'S, $\left.71^{\circ} 43^{\prime} \mathrm{W}\right)$ por el norte, hasta la península de Tongoy $\left(30^{\circ} 25^{\prime} \mathrm{S}, 71^{\circ} 51^{\prime} \mathrm{W}\right)$ por el sur. En el mes de enero de 1999 no hubo muestreo.

A los ejemplares se les registró su longitud total $\left(\mathrm{L}_{\mathrm{t}}\right)$ y peso total $\left(\mathrm{P}_{\mathrm{t}}\right)$, para establecer la relación talla-peso. Posteriormente se determinó el sexo y su estado de madurez sexual macroscópico, de acuerdo a lo propuesto por Flores \& Rojas (1985) para Polyprion oxygeneios, siendo posteriormente adaptada para G. nigra, de acuerdo a los caracteres tales como forma de la gónada, color, ubicación, relación con otros órganos, características de la membrana gonadal, presencia de irrigación sanguínea y porción ocupada por ésta en la cavidad abdominal. Las gónadas fueron pesadas en fresco en una balanza electrónica con precisión de $\pm 1 \mathrm{~g}$ y posteriormente fueron fijadas en formaldehído al 5\% para su análisis histológico. Se determinó la proporción de sexos total y mensual.

Para el análisis histológico se utilizó la técnica descrita por Oliva et al. (1986). Una vez efectuado los cortes histológicos, éstos fueron fotografiados y digitalizados para ser analizados en el programa computacional IMAGE-PRO, (Osorio \& Peniche-Ruiz 2001). Para determinar si existe diferencia en la maduración en distintos sectores de la gónada, se midieron 30 ovocitos de la moda más avanzada de la parte anterior, media y posterior de la gónada, con posterior análisis de varianza. 
Para caracterizar y describir los estados ovocitarios de acuerdo a sus características histológicas, se adoptó los criterios propuestos por Zanuy \& Carrillo (1973) y Cerisola et al. (1978), quienes se basaron en la forma del ovocito y su núcleo, en la disposición, tamaño y número de los nucléolos, en la afinidad por tinciones, en el vitelo primario y secundario, y en la morfología de la zona pelúcida (o radiata) y células foliculares. Los cortes histológicos fueron teñidos con hematoxilina de Harris y eosina alcohólica para su observación en microscopía de luz.

El ciclo reproductivo y la época de desove fueron determinados por métodos directos e indirectos. Entre los métodos directos, se estimó el índice gonadosomático:

$$
I G S=\frac{P_{g}}{P_{t}-P_{g}} * 100
$$

índice gonádico:

$$
I G=\frac{P_{g}}{L_{t}{ }^{3}} * 10000
$$

y factor de condición corregido:

$$
K_{c}=\frac{P_{t}-P_{g}}{L_{t}^{3}} * 100
$$

El análisis de estos índices obvia la subjetividad en la asignación de un estado de madurez macroscópico y reflejan con más fidelidad el proceso de maduración gonadal (Nikolsky 1963), además han sido utilizados para la descripción de ciclos reproductivos, en distintas especies y autores (Ramos 1982, Flores \& Rojas 1985, Alarcón \& Arancibia 1993, Chong \& González 1995, Leal \& Oyarzún 2003). Como método indirecto se utilizó el porcentaje de los estados de madurez sexual macroscópica (EMS) en forma mensual, analizando preferentemente la época de desove desde julio hasta diciembre.

Para determinar la talla de primera madurez sexual, tanto en machos como en hembras, se utilizaron dos metodologías. La primera descrita y utilizada por Arancibia et al. (1994), Chong \& González (1995), Leal \& Oyarzún (2003), entre otros, basada en el incremento relativo del índice gonadosomático (IR.IGS), así, la talla de primera madurez sexual es aquella donde existe el mayor incremento del IGS entre grupos de tallas correlativas y viene dada por la expresión:

Ihttp:// www. curveexpert. net

$$
I R \cdot I G S=\frac{\left(I G S_{j+1}-I G S_{j}\right)}{I G S_{j}} * 100
$$

donde IGS $_{j+1}$ es el IGS promedio de la clase de talla $j+1$ e IGS es el IGS promedio de la clase de talla $j$. La segunda metodología, se basa en el criterio del $50 \%$ de los ejemplares que muestran indicios de madurez reproductiva (EMS 3, 4 y 5) (Alarcón \& Arancibia 1993, Cubillos et al. 1999, Leal \& Oyarzún 2002), determinando el modelo logístico respecto a los porcentajes de ejemplares reproductivos por talla, dado por la formula:

$$
Y=\frac{a}{1+b^{*} e^{-c x}}
$$

donde $\mathrm{Y}$ es el porcentaje de ejemplares reproductivos, $\mathrm{x}$ es $\mathrm{L}_{\mathrm{t}} \mathrm{y}$ a, b y c son constantes; el modelo de ajuste se realizó con la ayuda del programa computacional Curve Expert $^{1}$.

\section{Resultados}

En el período de estudio se capturó un total de 251 ejemplares de G. nigra, los meses con mayor captura fueron agosto, septiembre, octubre, noviembre y marzo, con valores por sobre el $10 \%$ del total de peces muestreados, los meses de mayor captura de hembras fueron septiembre, noviembre y marzo, mientras que machos, agosto, septiembre, octubre y noviembre, en todos estos meses la captura para ambos sexos fue superior al $10 \%$ del total por género capturado (Fig. 1). La proporción de machos y hembras fue de un $45 \%$ y $55 \%$ respectivamente. En la captura mensual por lo general aparecieron más hembras que machos, sin embargo, en agosto, septiembre y octubre esta relación fue inversa.

La relación talla-peso para hembras estuvo dada por la relación $\mathrm{P}_{\mathrm{t}}=0,0255 * \mathrm{~L}_{\mathrm{t}}{ }^{2,953}$, mientras que en machos fue de $\mathrm{P}_{\mathrm{t}}=0,0124 * \mathrm{~L}_{\mathrm{t}}{ }^{3,0927}$, no existiendo diferencias significativas entre ambos modelos (ANDEVA; $\mathrm{F}_{(1,210)} ; P$ $=0,839$, Fig. 2).

La histología del ovario mostró diferentes estados ovocitarios al interior de la gónada en EMS 3, donde el diámetro medio de los ovocitos en la parte anterior, media y posterior, fue de 504; 516 y $519 \mu \mathrm{m}$ respectivamente, no encontrando diferencias significativas en el tamaño de estos ovocitos (ANDEVA, $\mathrm{F}_{(2,87)} ; P=0,4269$ ).

La descripción de los estados ovocitarios para las gónadas femeninas de Graus nigra, se indica a continuación: 


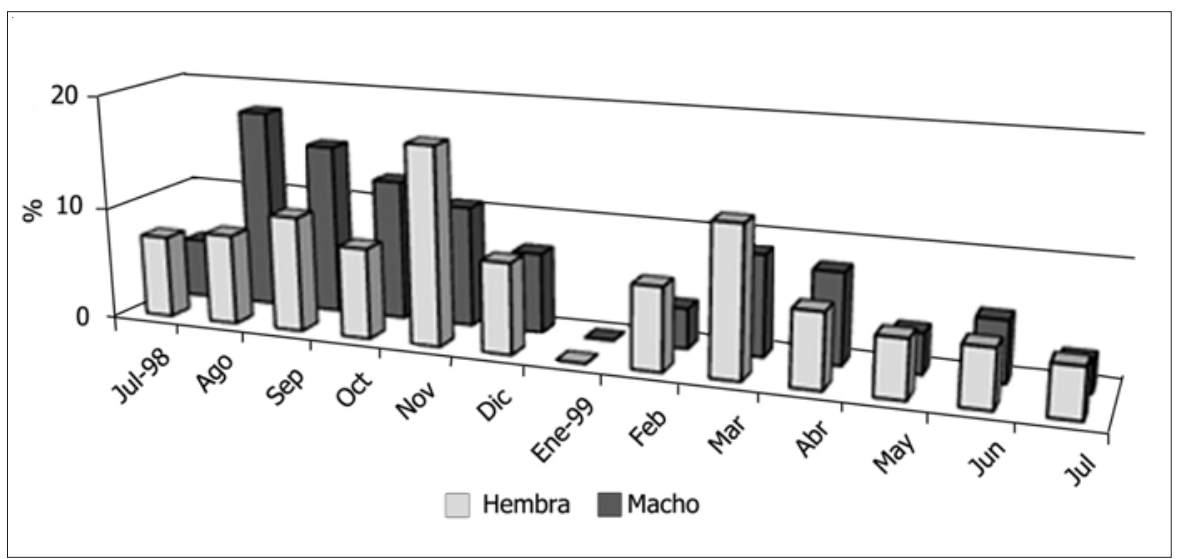

Figura 1. Porcentaje de ejemplares capturados de Graus nigra por sexo y mes de muestreo / Graus nigra specimen captured for sex and month of sampling

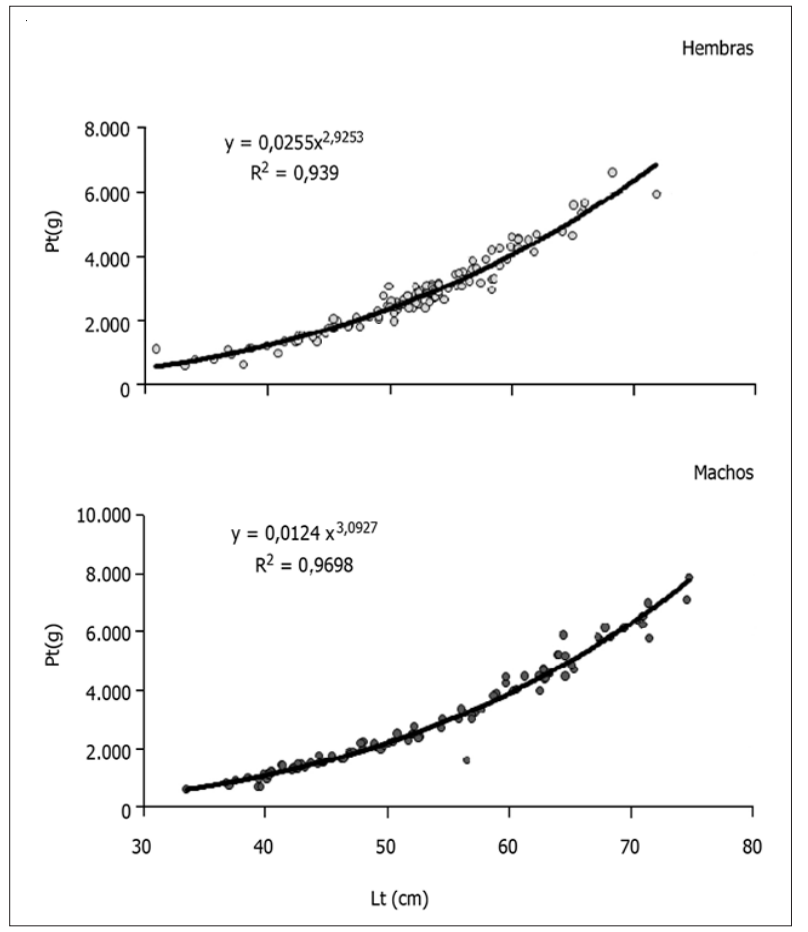

Figura 2. Relación talla-peso para hembras y machos de Graus nigra / Size-weight relationship for females and males of Graus nigra

Oogonias: Ligeramente ovoides alcanzando tamaños que fluctúan entre los 5,5 y 8,5 $\mu \mathrm{m}$ de diámetro. Se pueden encontrar solas o agrupadas alrededor de lamelas ovígeras, dando origen a los nidos ovígeros, que en la tinción se ven muy claros, casi transparentes. El núcleo es esférico y tiene una posición central, rodeado por el escaso citoplasma acidófilo. No se observa un límite celular neto, ni tampoco estructuras foliculares rodeando las ovogonias (Fig. 3).
Oocito I (OoI): Perinucleolar temprano, de forma esférica o ligeramente ovoide. Mide entre 91 y $139 \mu \mathrm{m}$ de diámetro. El citoplasma es altamente basófilo y homogéneo. El núcleo (vesícula germinal) es central y ovoide, con límites bien definidos, mide entre 32 y $66 \mu \mathrm{m}$ y puede presentar algunos corpúsculos basófilos en su interior. Los nucléolos son esféricos u ovoides de 4 a $7 \mu \mathrm{m}$ de diámetro, su número varía de 1 a 4 nucléolos periféricos en las células de menor tamaño y hasta 8 en los de mayor tamaño. Este ovocito no presenta envolturas ovocitarias ni células foliculares (Fig. 4).

Oocito II (OoII): Perinucleolar tardío. El ovocito se encuentra rodeado por una delgada capa de células foliculares aplanadas con límites difusos. Estos ovocitos previtelogénicos son ovoides y miden entre 174 y $215 \mu \mathrm{m}$ de diámetro. El citoplasma es homogéneo y menos basófilo que en el estado anterior, con vacuolas o vesículas esféricas alrededor del núcleo, que corresponden al vitelo primario. El núcleo varía de ovoide a esférico, tiene una disposición central con límites algo irregulares, midiendo entre $95 \mathrm{y}$ $110 \mu \mathrm{m}$. El núcleo puede llegar a contener hasta 14 nucléolos esféricos y periféricos que miden entre 9 y 12 $\mu \mathrm{m}$, presentando además algunos corpúsculos basófilos (Fig. 5).

Oocito III (OoIII): Vitelogénico temprano. Los ovocitos miden entre 282 y $315 \mu \mathrm{m}$ de diámetro. El citoplasma cortical es basófilo y homogéneo, mientras que alrededor del núcleo es de menor intensidad basofílica. Numerosas vacuolas rodean al núcleo, el que alcanza un tamaño entre 96 y $100 \mu \mathrm{m}$ de diámetro y contiene numerosos nucléolos en posición periférica, lo cual hace que el núcleo presente límites muy irregulares. En este estado aparecen los primeros gránulos de vitelo en el citoplasma y los primeros 
primordios de las envolturas ovocitarias. Es posible distinguir una zona pelúcida o radiata, como una monocapa lisa de 9 a $16 \mu \mathrm{m}$ de espesor, altamente basófila. Externamente al ovocito se encuentra una zona folicular formada por una monocapa de células granulosas aplanadas y con núcleos ovoides altamente basófilos (Fig. 6).

Oocito IV (OoIV): Vitelogénico tardío. Los ovocitos son ovoides y su tamaño fluctúa entre los 320 y $415 \mu \mathrm{m}$ de diámetro. La presencia de gránulos de vitelo en el citoplasma es mayor, pero en la zona cortical hay una delgada capa de citoplasma homogéneo altamente basófilo. El núcleo es central y esférico, con límites irregulares y con un diámetro que fluctúa entre 90 y $110 \mu \mathrm{m}$. El material nuclear es acidófilo y homogéneo, con algunos corpúsculos basófilos y con nucléolos esféricos y periféricos de 15 a 20 $\mu \mathrm{m}$ de diámetro. La zona pelúcida se engruesa, midiendo entre 5,5 y $10 \mu \mathrm{m}$ de espesor. Esta zona está formada por una bicapa bien diferenciada; la monocapa interna es estriada y un poco más gruesa que la externa. Externamente en el epitelio folicular, que estaba compuesto en el estado anterior (OoIII) solo por células granulosas, aparece una delgada y difusa capa de células tecales (Fig. 7).

Oocito V (OoV): Maduro. Ovocitos de forma esférica a ovoide, que alcanzan a medir entre 429 y $576 \mu \mathrm{m}$ de diámetro. El citoplasma está constituido por gránulos grandes y esféricos de vitelo, pudiendo observarse en algunos de los ovocitos vacuolas centrales con algo de citoplasma basófilo. Algunos ovocitos presentan núcleos dendriformes de 184 a $218 \mu \mathrm{m}$ de diámetro, pero en la mayoría no es posible observarlos. Las envolturas foliculares están formadas por dos tipos de células: las tecales y las granulosas. Las primeras son externas y muy aplanadas, con núcleos grandes y aplanados que ocupan gran parte del citoplasma. Las células granulosas (internas) son notablemente más esféricas, de límites difusos y algo más acidófilas que las tecales. La zona pelúcida se engruesa, alcanzando aproximadamente de 6,5 a $15,5 \mu \mathrm{m}$. Por su patrón de coloración, es posible diferenciar dos regiones: una interna, estriada y ancha, de tonalidad más clara (acidófila), y otra externa, de coloración más obscura (basófila) y delgada, llegando a ser esta última $1 / 3$ de la zona pelúcida total (Fig. 8).

Oocito VI (OoVI): Hidratado. Estos ovocitos alcanzan tamaños superiores a los $700 \mu \mathrm{m}$ de diámetro. Generalmente colapsan al ser procesados histológicamente, razón por la cual no pudieron ser observados.

El análisis del IGS e IG indicó que la época reproductiva en esta especie, tanto para hembras como machos fue entre

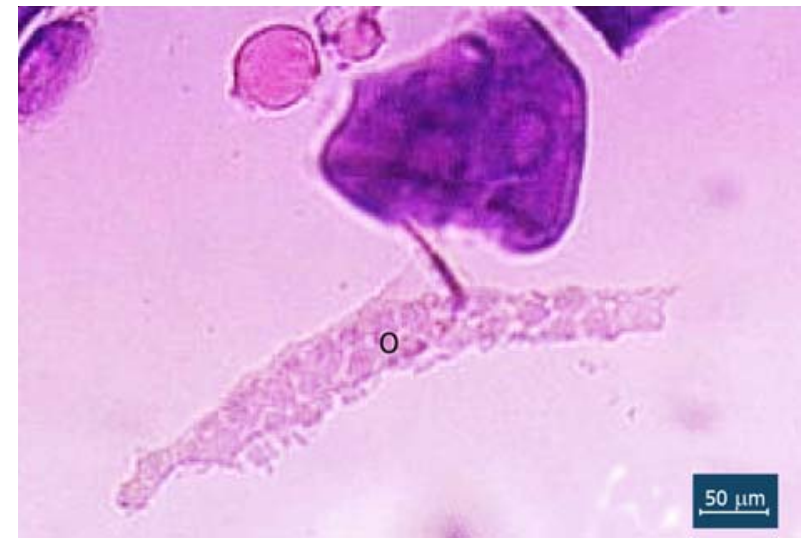

Figura 3. Corte histológico de la gónada femenina de Graus nigra, se destaca la presencia de oogonias (0) / Histological cut of female gonad of Graus nigra, the presence of oogonias (0) is highlighted

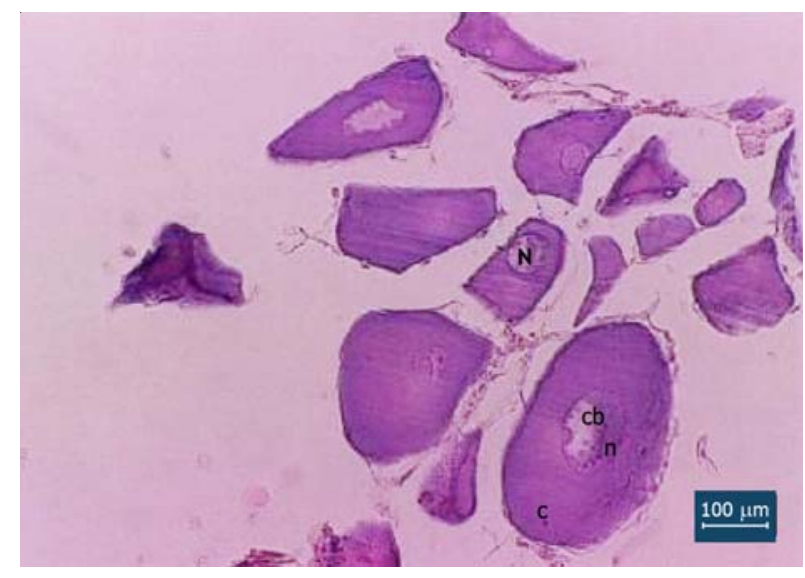

Figura 4. Oocito I, perinuclear temprano en corte histológico de gónada de Graus nigra. $\mathrm{N}=$ núcleo, $\mathrm{n}=$ nucléolo, $\mathrm{c}=$ citoplasma, cb=corpúsculos basófilos / Oocyte I, early perinuclear in histological cut of Graus nigra gonad. N=nucleus, $n=$ nucleoli, $c=c i t o p l a s m$, $\mathrm{cb}=$ corpuscle basophil

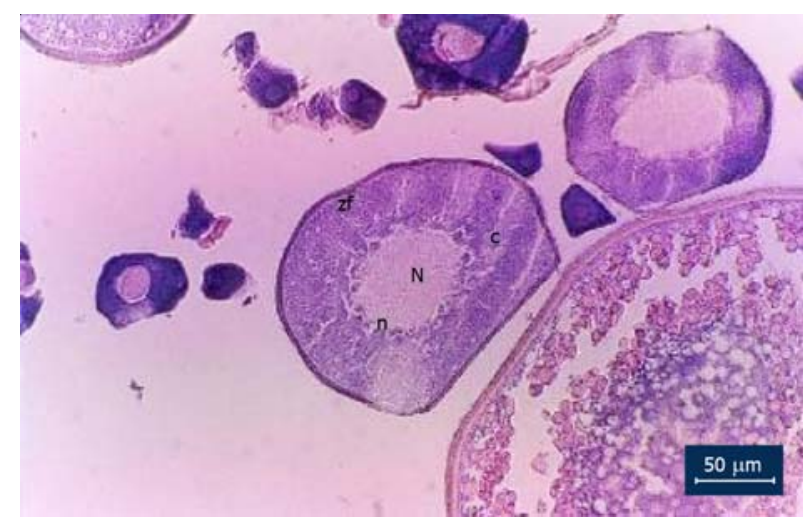

Figura 5. Oocito II, perinuclear tardío en corte histológico de gónada de Graus nigra. $\mathrm{N}=$ núcleo, $\mathrm{n}=$ nucléolo, $\mathrm{c}=$ citoplasma, $\mathbf{z f}=\mathbf{z o n a}$ folicular / Oocyte II, late perinuclear in histological cut of Graus nigra gonad. $N=n u c l e u s, n=$ nucleoli, $c=c i t o p l a s m, z f=f o l l i c u l a r$ zone 


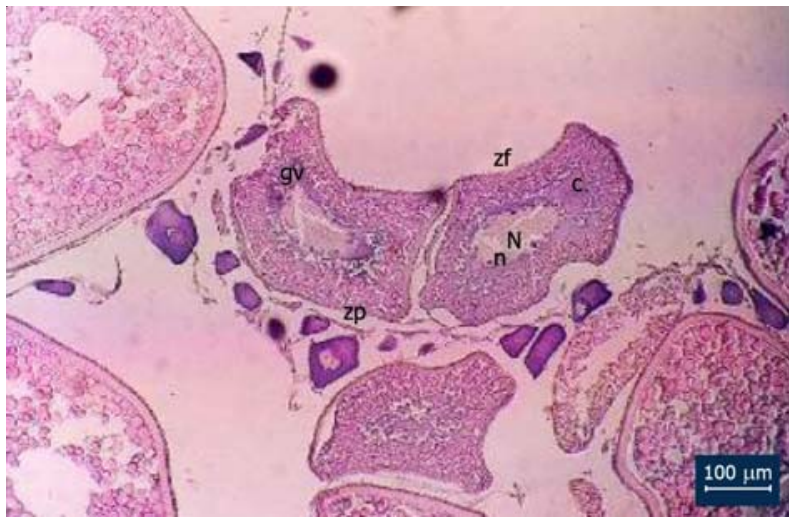

Figura 6. Oocito III, vitelogénico temprano en corte histológico de gónada de Graus nigra. $\mathrm{N}=$ núcleo, $\mathrm{n}=$ nucléolo, $\mathrm{c}=$ citoplasma, $\mathbf{z f}=\mathbf{z o n a}$ folicular, $\mathbf{z p = z o n a}$ pelúcida, $\mathbf{g v}=$ granulos de vitelo / Oocyte III, early vitellogenic in histological cut of Graus nigra gonad. $\mathrm{N}=$ nucleus, $\mathrm{n}=$ nucleoli, $\mathrm{c}=$ citoplasm, $\mathrm{z} f=$ follicular zone, $\mathrm{z} p=$ pellucida zone, gv=vitello granules

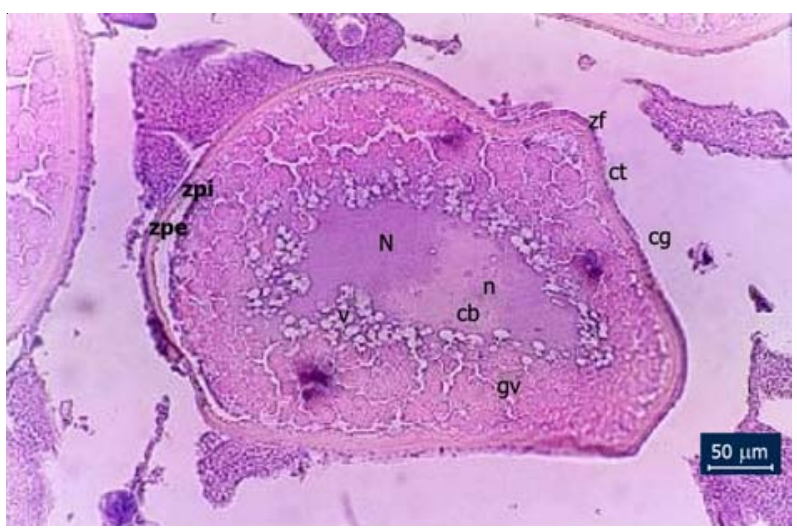

Figura 7. Oocito IV, vitelogénico tardío en corte histológico de gónada de Graus nigra. $\mathrm{N}=$ núcleo, $\mathrm{n}=$ nucléolo, $\mathrm{c}=$ citoplasma, $\mathrm{cb}=$ corpúsculos basófilos, $\mathrm{cg}=$ células granulosas, $\mathrm{ct}=$ =élulas tecales, v=vacuolas, $z$ pe=zona pelúcida externa, $z \mathbf{p i = z o n a}$ pelúcida interna, gv=granulos de vitelo / Ooyte IV, late vitellogenic in histological cut of Graus nigra gonad. N=nucleus, $n=$ nucleoli, $c=c i t o p l a s m, c b=$ basophil corpuscle, $c g=$ granule cells, $c t=$ thecacells, $v=$ vacuole, $z p e=e x t e r n a l$ pellucida zone, $z p i=$ internal pellucida zone, gv=vitello granules

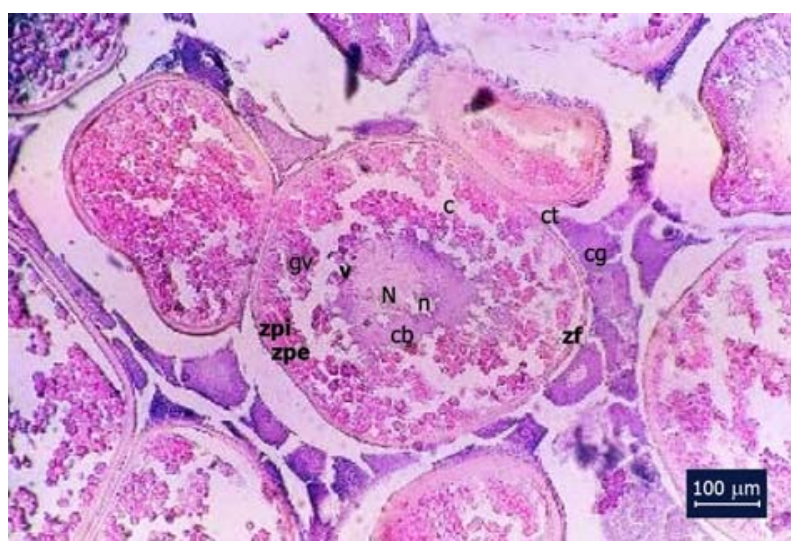

julio y diciembre, periodo de invierno donde se produce la madurez gonadal preferentemente, ocurriendo los desoves en primavera (Fig. 9).

El índice de condición (K) mostró una disminución para los meses de invierno (julio a septiembre), especialmente en las hembras, producto del proceso fisiológico que ocurre en la gónada como es la vitelogénesis (Fig. 9), situación que no se observó tan claramente en machos.

Para hembras y machos de G. nigra se describieron las características de la gónada en sus diferentes estados de madurez sexual (EMS), (Tabla 1). En agosto, tanto para hembras y machos, comienzan a aparecer los EMS3, en septiembre, octubre y noviembre los EMS4, visualizándose gónadas desovadas desde octubre en adelante (Fig. 10).

La talla de primera madurez sexual determinada por el criterio del $50 \%$ de los ejemplares reproductivos, se estimó para hembras en 39,5 cm (Fig. $11 \mathrm{~A}$ ) y para machos en 42,7 $\mathrm{cm}$ (Fig. $11 \mathrm{~B}$ ). Por el método del IR.IGS se estimó una talla de primera madurez para hembras de $40,8 \mathrm{~cm}$ (Fig. 12 A) y de $40,5 \mathrm{~cm}$ para machos (Fig. $12 \mathrm{~B}$ ).

\section{Discusión}

Generalmente en las especies de peces la proporción de sexos es 1:1, así lo demuestran estudios realizados en Polyprion oxygeneios (Flores \& Rojas 1985) y en Lutjanus guttatus (Arellano et al. 2001). En agosto, septiembre y octubre se observó una mayor presencia de machos, no se dispone de antecedentes formales, pero esta situación podría responder a la conducta de las hembras, que permanecen refugiadas en grietas y cuevas durante el periodo de desove, no siendo visible con facilidad para sus captores. En el resto de los meses la proporción de hembras siempre fue mayor que la de los machos. En el caso de Paralichthys microps en las costas de Concepción (Chong \& González 1995), las capturas representadas en su mayoría por hembras, podrían ser por una diferencia en el uso del ambiente, en relación a la batimetría o a una longevidad menor en los machos.

Figura 8. Oocito $\mathrm{V}$ maduro en corte histológico de gónada de Graus nigra. $\mathrm{N}=$ núcleo, $\mathrm{n}=$ nucléolo, $\mathrm{c}=$ citoplasma, $\mathrm{cb}=$ corpúsculos basófilos, $\mathbf{c g}=$ células granulosas, $\mathrm{ct}=$ =élulas tecales, $\mathrm{v}=$ vacuolas, $\mathbf{z f}=\mathbf{z o n a}$ folicular, zpe=zona pelúcida externa, zpi=zona pelúcida interna, gv=granulos de vitelo / Oocyte $V$ mature in histological cut of Graus nigra gonad. $\mathrm{N}=$ nucleus, $\mathrm{n}=$ nucleoli, $\mathrm{c}=$ citoplasm, $\mathrm{cb}=$ basophil corpuscle, $\mathrm{cg}=$ granule

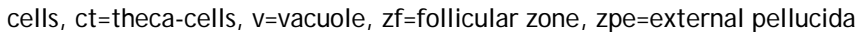
zone, zpi=internal pellucida zone, gv= vitello granules 


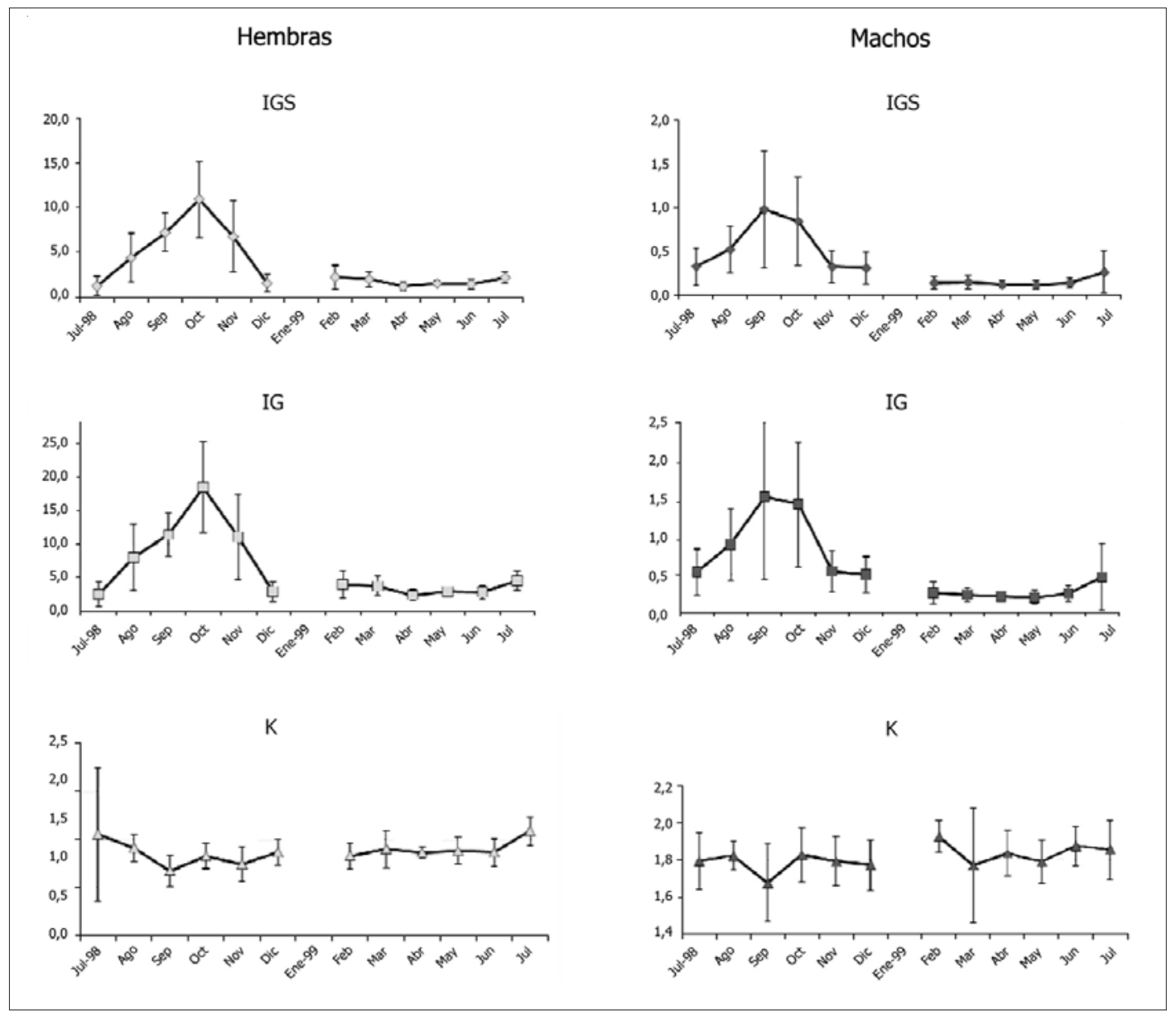

Figura 9. Indices gonadosomático (IGS), índice gonádico (IG) y de condición (K) en hembras y machos de Graus nigra / Gonadosomatic (IGS), gonadic index (IG) and condition index (K) in female and males of Graus nigra

Las constantes b de la relación talla-peso es concordante con los valores reportado para otras especies de la misma familia (Froser \& Pauly 2009).

Es recurrente encontrar en la literatura distintas denominaciones a los procesos de maduración de los ovarios respecto a los estados oocitarios, e.g., para $P$. microps Chong \& González (1995) describen los estados de inmaduro, previtelogénico, vitelogénico, maduro, hidratado y postovulatorio; para Sardinops sagax Herrera et al. (1994) describen los estados de inmaduro, parcialmente vitelado, vitelado, próximo a la hidratación, hidratado, en desove y desovado; para Lutjanus guttatus, Arellano et al. (2001) describen los estados de reposo, desarrollo, maduración, desove y postdesove; para Solea solea Ramos (1983) describe dos fases de crecimiento ovocitario, la primera con ovocitos carentes de vitelo y la segunda, con ovocitos con mayor o menor cantidad de vitelo. Es importante unificar la denominación de los distintos procesos de maduración gonadal.
Referente a los estados ovocitarios, G. nigra presenta oocitos similares a la mayoría de los teleósteos que liberan sus huevos al pélago, diferenciándose en el tamaño, tanto de los oocitos como de los distintos organelos (Ramos 1983; Vizziano \& Berois 1990 y Cortes 1998). Sin embargo, difiere consistentemente con aquellas especies que tienen distinto comportamiento reproductivo, como Sicyases sanguineus (Gobiesocidae) en el cual se ha observado una estructura de fijación denominada capa vellosa, provista de filamentos de fijación (Cerisola 1984), organelo que está ausente en $G$. nigra.

Los oocitos en estado hidratado, que no pudieron ser observados en G. nigra por colapsar las técnicas histológicas, han sido descritos para Sicyases sanguineus e Isacia conceptionis con formas estrelladas y muy irregulares, sin poder visualizar el núcleo celular (Cerisola 1984; Cortez 1998). Los folículos postovulatorios (FPO) y la atresia oocitaria, no se describen en este trabajo ya que no se consideran en sentido estricto como estados oocitarios, 
correspondiendo los primeros como aquellos folículos presentes después de haberse realizado un desove, y que muchos autores los utilizan para determinar en forma más precisa este estado (Herrera et al. 1994, Chong \& González 1995, Arellano et al. 2001). La atresia ovocitaria es el proceso por el cual los ovocitos no desovados son reabsorbidos, y la proporción de estos dentro de la gónada fue utilizada por algunos autores para estudios más acabados sobre fecundidad (Balbontín \& Fischer 1981).

La presencia de diferentes estados ovocitarios dentro de la gónada en EMS 3 indicó que G. nigra tiene una maduración gonadal asincrónica y sería un desovador parcial dentro de un período reproductivo.
En especies de latitudes altas, donde el período estival es corto, se observó que las especies de peces presentan generalmente un período de desove reducido, definido y de desove total (Cushing 1975, Blaxter \& Hunter 1982). A medida que se avanza hacia latitudes bajas (zonas subtropical y tropical), el período de reproducción es más prolongado, puede limitarse a una temporada más amplia, pero definida con desoves parciales o puede durar todo el año (Cushing 1975). G. nigra presenta un tipo de reproducción correspondiente a especies de zonas subtropicales, con un marcado período de actividad reproductiva correspondiente a primavera y a un desovante parcial.

Tabla 1. Estados de madurez sexual (EMS) macroscópico para hembras y machos de Graus nigra / Macroscopic sexual maturity stages (EMS) for females and males of Graus nigra

\begin{tabular}{|c|c|c|}
\hline ESTADO & HEMBRA & MACHO \\
\hline \multirow[t]{2}{*}{ 1. Juvenil y/o virginal } & \multicolumn{2}{|c|}{$\begin{array}{l}\text { Órganos sexuales muy pequeños, ubicados bajo la columna vertebral en el tercio posterior; } \\
\text { gónadas cristalinas, transparentes de un amarillo incoloro }\end{array}$} \\
\hline & $\begin{array}{l}\text { Ovarios pequeños, hasta } 4 \mathrm{~cm} \text { de longitud y } 1 \mathrm{~cm} \\
\text { de alto. Ocupan cerca de un cuarto de la cavidad } \\
\text { abdominal. Su forma parece un pequeño saco } \\
\text { alargado, con un color amarillo translúcido, aún no } \\
\text { se aprecian los vasos capilares. Los oocitos son } \\
\text { imperceptibles a ojo desnudo }\end{array}$ & $\begin{array}{l}\text { Testículos pequeños, no superan los } 5 \mathrm{~cm} \text { de longitud } \\
\text { son muy delgados, como máximo un par de mm de } \\
\text { alto. Ocupan un poco menos de un tercio de la } \\
\text { cavidad abdominal. En sección transversal su forma } \\
\text { es similar a la de un triángulo isósceles de gran } \\
\text { hipotenusa. La coloración es de blanquecino opaca a } \\
\text { café claro }\end{array}$ \\
\hline \multirow[t]{2}{*}{ 2. Reposo } & \multicolumn{2}{|c|}{$\begin{array}{l}\text { Las gónadas son opacas y el sistema vascular se encuentra desarrollado alrededor de ella y visible } \\
\text { claramente }\end{array}$} \\
\hline & $\begin{array}{l}\text { Ovarios ocupan aproximadamente la mitad de la } \\
\text { longitud de la cavidad abdominal, la membrana } \\
\text { ovárica es de un color blanco translucido opaco }\end{array}$ & $\begin{array}{l}\text { Testiculos ocupan poco más de la mitad de la } \\
\text { longitud de la cavidad abdominal. Son de una } \\
\text { tonalidad blanca opaca }\end{array}$ \\
\hline 3. En Maduración & $\begin{array}{l}\text { Ovarios grandes, ocupan cerca de la mitad de la } \\
\text { cavidad abdominal, voluminosos y turgentes. } \\
\text { Tienen una tonalidad amarilla y los oocitos son } \\
\text { visibles a ojo desnudo. Abundante irrigación } \\
\text { sanguínea }\end{array}$ & $\begin{array}{l}\text { Testículos relativamente grandes de una longitud de } \\
\text { la mitad de la cavidad abdominal. Presentan lóbulos } \\
\text { turgentes de una tonalidad blanquecina. La irrigación } \\
\text { sanguínea es desarrollada }\end{array}$ \\
\hline 4. Maduro & $\begin{array}{l}\text { Ovario de grandes dimensiones, ocupan gran parte } \\
\text { de la cavidad abdominal. Turgentes, con una } \\
\text { membrana ovárica translúcida que deja ver los } \\
\text { oocitos algunos hidratados distribuidos } \\
\text { homogéneamente en la totalidad de la gónada. Son } \\
\text { de una coloración amarillenta y poseen gran } \\
\text { irrigación sanguínea }\end{array}$ & $\begin{array}{l}\text { Testículos desarrollados. Ocupan más de la mitad de } \\
\text { la longitud de la cavidad abdominal. Presentan } \\
\text { lóbulos turgentes de una coloración blanquecina. } \mathrm{Al} \\
\text { presionar el abdomen escurre un líquido viscoso de } \\
\text { color blanco }\end{array}$ \\
\hline 5. Desovado & $\begin{array}{l}\text { Ovarios flácidos, tienen apariencia de bolsas } \\
\text { vacías; al cortarlas en forma trasversal se puede } \\
\text { observar un lumen amplio. La membrana ovárica se } \\
\text { engruesa y se pone más opaca, donde se aprecian } \\
\text { zonas hemorrágicas. }\end{array}$ & $\begin{array}{l}\text { Testículos pierden turgencia, son más opacos y } \\
\text { presentan zonas hemorrágicas }\end{array}$ \\
\hline
\end{tabular}




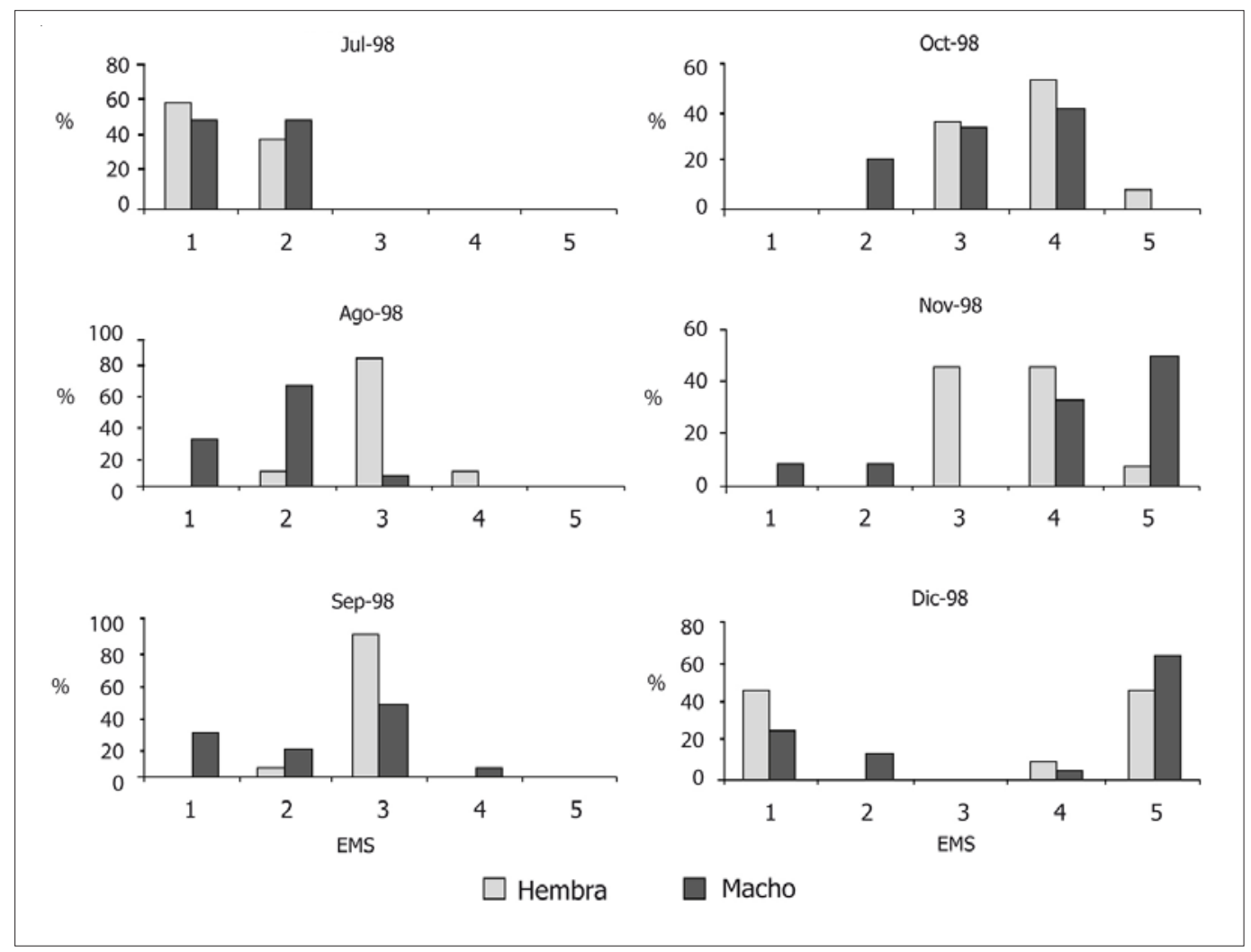

Figura 10. Estados de madurez sexual (EMS) en ejemplares de Graus nigra durante el periodo reproductivo / Sexual maturity stages (EMS) in Graus nigra specimen during the reproductive period

Los mayores valores promedios mensuales del IGS e IG, tanto para machos como para hembras, ocurren en los meses de primavera, corroborándose esta máxima actividad reproductiva con los porcentajes de estados de madurez sexual macroscópicos.

La talla de primera madurez sexual determinada para hembras por el método del $50 \%$ de ejemplares reproductivos $(39,5 \mathrm{~cm})$ difiere de la obtenida mediante el método del IR.IGS $(40,8 \mathrm{~cm})$, esta última fue un $3,3 \%$ superior. En machos también hay una diferencia entre ambos métodos analizados (42,7 y 40,5 cm), donde el método del IR.IGS fue inferior al del $50 \%$ de ejemplares maduros en un $5,1 \%$. La diferencia entre ambos métodos también se presenta en otros recursos ícticos como en Brama australis (Lael \& Oyarzún 2003), donde hay una mayor talla de primera madurez sexual por el método del IR.IGS. Es importante indicar que esta metodología es muy sensible al tamaño de la muestra analizada, que en el caso de peces del litoral rocoso, su captura y baja abundancia es una complicación.

Los juveniles de G. nigra reclutan en pozas litorales. Berrios \& Vargas (2000) verifican la existencia de juveniles en la zona norte del país en las estaciones de invierno y primavera, posibles reclutas del evento reproductivo del año anterior. Si el período reproductivo transcurre desde agosto hasta diciembre, los huevos y larvas estarían presentes en el pélago durante el verano y otoño para posteriormente aventurarse en las pozas intermareales, antecedentes al respecto no se disponen para Graus nigra, sólo el registro de larvas de Kyphosidae frente a la costa de Concepción (Landaeta et al. 2008), que podrían corresponder a esta especie, según lo propuesto por Vargas \& Pequeño 2004, siendo G. nigra una especie Kyphosidae que se distribuye más australmente en las costas de Chile y las otras especies de la familia tienen distribución insular o continental más al norte (Pequeño 2004, Pequeño \& Sáez 2000, 2008).

La pesquería de G. nigra se encuentra en un régimen de libre acceso, no existiendo para esta especie y para todos los recursos ictiológicos conocidos como "peces de roca", una regulación pesquera o restricciones para su captura (Pequeño \& Olivera 2005, Perez-Matus \& Buschmann 2003), condición que fue destacada para este recurso por Vargas \& Pequeño (2004). En la actualidad hay síntomas claros de sobreexplotación en algunas especies de peces 


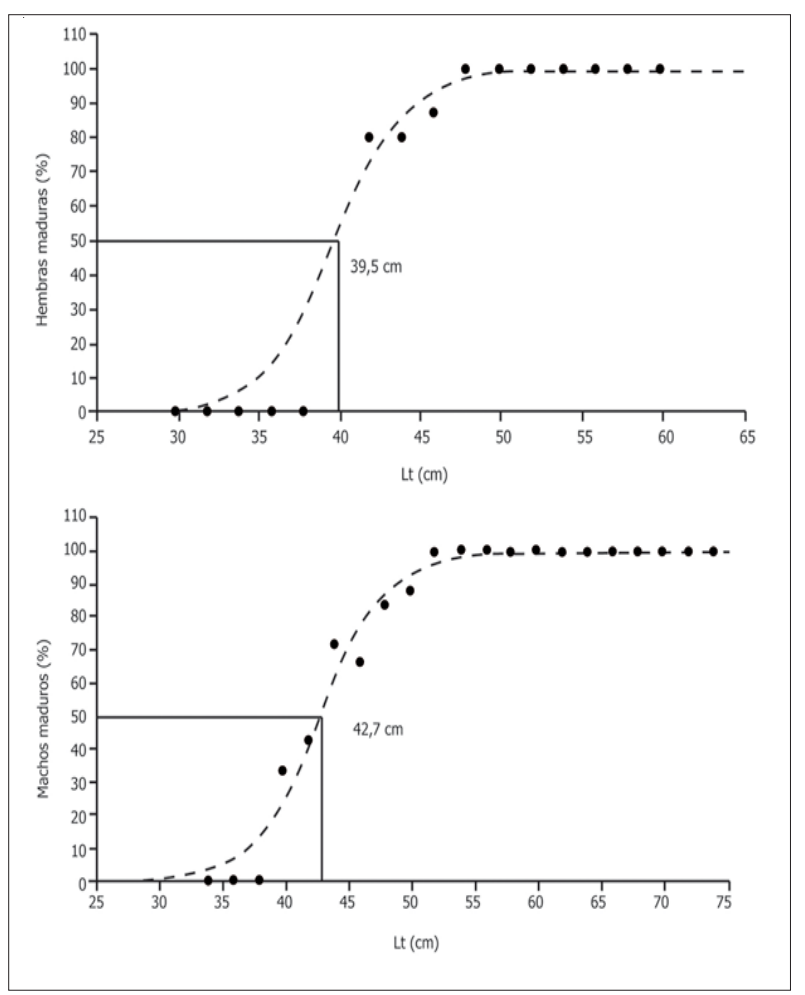

Figura 11. Talla de primera madurez sexual en hembras (A) y machos (B) de Graus nigra, determinada por el método del $\mathbf{5 0 \%}$ de ejemplares maduros / Size of first sexual maturity in females (A) and males (B) of Graus nigra, determined by the method of $50 \%$ of mature specimens

litorales de roca, donde la implementación de las AMERB ha tenido efectos positivos sobre la abundancia y riqueza de estas especies (Godoy 2008).

En la actualidad no se cuenta con un registro de las tallas de los peces capturados con fines comerciales. Esta información permitiría conocer la fracción de peces que estaría siendo capturada bajo la talla de primera madurez y acercarnos a estimar el impacto de la actividad pesquera en este recurso. En los mercados se puede observar con cierta frecuencia ejemplares de G. nigra de pesos cercanos a 1,0 $\mathrm{kg}$, tamaños que estarían bajo la talla de primera madurez. Las asociaciones de buceo deportivo, conscientes de esta situación han establecido como peso mínimo de extracción los $2 \mathrm{~kg}$.

Se hace necesario regular la extracción de esta especie con capturas en tallas superiores a los $40 \mathrm{~cm}$ de $\mathrm{L}_{\mathrm{t}} \mathrm{y} / \mathrm{o}$ de un $P_{t}$ superior a $1,5 \mathrm{~kg}$. Favorecería positivamente si junto a lo anterior, se pudiera aplicar una veda extractiva entre agosto y noviembre, periodo reproductivo determinado en la presente investigación.

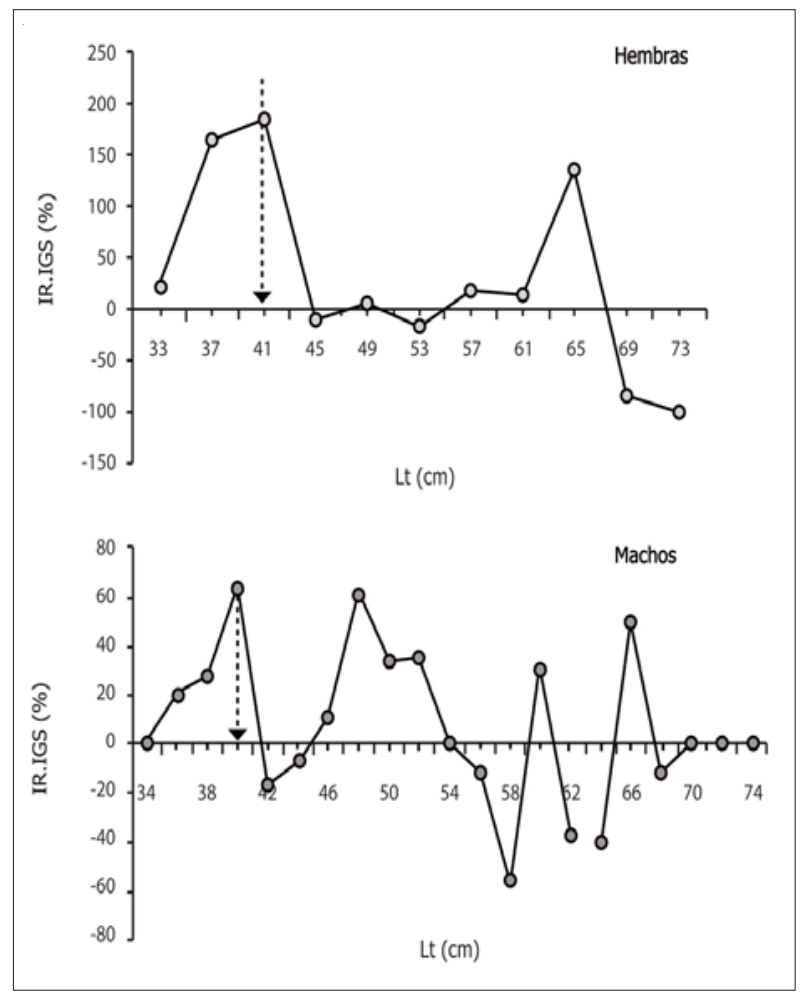

Figura 12. Talla de primera madurez sexual en hembras (A) y machos (B) de Graus nigra, determinada por el método del incremento relativo del IGS / Size of first sexual maturity in females (A) and males (B) of Graus nigra, determined by the method of the relative increment of the IGS

\section{Agradecimientos}

Se agradece a la Dra. (c) Patricia Zarate y al Dr. Benjamín Ruttenberg de la Universidad de Florida por su colaboración en la redacción del abstract, al Dr. (c) Alonso Vega de la Universidad Católica del Norte por su apoyo en los análisis estadísticos y al Dr. Ciro Oyarzún por sus comentarios y aportes.

\section{LITERATURA CITADA}

Alarcón R \& H Arancibia. 1993. Talla de primera madurez sexual y fecundidad parcial en la merluza común, Merluccius gayi gayi (Guichenot, 1814). Ciencia y Tecnología del Mar 16: 33-45.

Angeles B \& J Mendo. 2005. Crecimiento, fecundidad y diferenciación sexual del lenguado Paralichthys adspersus (Steindachner) de la costa central del Perú. Ecología Aplicada 4(1/2): 105-112.

Arancibia H, L Cubillos, J Remmaggi \& R Alarcón. 1994. Determinación de la talla de madurez sexual y fecundidad parcial de la sardina común, Strangomera bentincki (Norman, 1936), del área de Talcahuano, Chile. Biología Pesquera 22: 11-17. 
Arellano-Martinez A, A Rojas-Herrera, F García-Dominguez, B Ceballos-Vasques \& M Villalejo-Fuerte. 2001. Ciclo reproductivo del pargo lunarejo Lutjanus guttatus (Steindachner, 1869) en las costas de Guerrero, México. Revista de Biología Marina y Oceanografía 36(1): 1-8.

Balbontín F \& W Fischer. 1981. Ciclo sexual y fecundidad de la merluza Merluccius gayi gayi, en las costas de Chile. Revista de Biología Marina 17(3): 285-334.

Berrios V \& M Vargas. 2000. Estructura del ensamble de peces intermareales de la costa rocosa del norte de Chile. Revista de Biología Marina y Oceanografía 35(1): 73-81.

Blaxter JHS \& JR Hunter. 1982. The biology of the clupeoid fishes. Advances in Marine Biology 20: 1-233.

Cerisola H. 1984. Cambios estructurales del folículo ovárico durante su maduración en el pejesapo Sicyases sanguineus, Muller y Troschel 1843 (Gobiesocidae: Teleostei). Revista de Biología Marina 20(1): 1-21.

Cerisola H, F Grisolía \& E Méndez. 1978. Aspectos estructurales y ultraestructurales del ovario del pejerrey de agua dulce Basilichthys australis Eigenmann. Anales del Museo de Historia Natural de Valparaíso 11: 175-190.

Chong J \& P González. 1995. Ciclo reproductivo del lenguado de ojos chicos, Paralichthys microps (Günther, 1881) (Pleuronectiformes, Paralichthyidae) frente al litoral de Concepción, Chile. Biología Pesquera 24: 39-50.

Claramunt G, G Herrera \& P Pizarro. 1994. Producción potencial anual de huevos por tallas en Sardinops sagax (Jenysns, 1842) del norte de Chile. Revista de Biología Marina 29(2): 211-233.

Cordo H, L Machinandiarena, G Macchi \& MF Villarino. 2002. Talla de primera madurez del abadejo (Genypterus blacodes) en el Atlántico sudoccidental. Informe Técnico, INIDEP 47: 1-16.

Cortez N. 1998. Dinámica ovárica, época de desove y fecundidad parcial de la "cabinza" Isacia conceptionis (Perciformes, Haemulidae) en la bahía la Herradura, IV Región, Coquimbo. Tesis de Biología Marina, Facultad de Ciencias del Mar, Universidad Católica del Norte, Coquimbo, 75 pp.

Cushing DH. 1975. Ecología marina y pesquerías, 252 pp. Editorial Acribia, Zaragoza.

Flores H \& P Rojas. 1985. Talla de primera madurez, época de desove y fecundidad del bacalao de Juan Fernández Polyprion oxygeneios (Bloch y Schneider, 1801) (Pisces: Percichthyidae). En: Arana P (ed). Investigaciones marinas en el Archipiélago de Juan Fernández, pp. 311-322. Escuela de Ciencias del Mar, Universidad Católica de Valparaíso, Valparaíso.

Froese R \& D Pauly. 2009. FishBase. [on-line] <http:// www.fishbase.org>

Fuentes H. 1982. Feeding habits of Graus nigra (Labridae) in coastal waters of Iquique in northen Chile. Japanese Journal of Ichthyology 29(1): 95-98.

Godoy N. 2008. Pesca por buceo de peces litorales de roca: desembarques, composición de las capturas y efectos sobre la riqueza y la abundancia de las especies. Tesis de Magíster en Ciencias del Mar, Universidad Católica del Norte, Coquimbo, 75 pp.

Hernández CE, PE Neill, JM Pulgar, FP Ojeda \& F Bozinovic. 2002. Water temperature fluctuations and territoriality in the intertidal zone: two possible explanations for the elevational distribution of body size in Graus nigra. Journal of Fish Biology 61: 472-488.

Herrera G, G Claramunt \& P Pizarro. 1994. Dinámica ovárica de la sardina española (Sardinops sagax) del norte de Chile, periodo abril 1992- marzo 1993. Análisis por estrato de talla. Revista de Biología Marina 29(2): 147-166.

Johnson GD \& RA Fritzsche. 1989. Graus nigra an omnivorous girellid, with a comparative osteology and comments on relationships of the Girellidae (Pisces: Perciformes). Proceedings of the Academy of Natural Sciences of Philadelphia 141: 1-27.

Landaeta MF, R Veas, J Letelier \& L Castro. 2008. Larval fish assemblages off central Chile upwelling ecosystem. Revista de Biología Marina y Oceanografía 43(3): 569-584.

Leal E \& C Oyarzún. 2003. Talla de madurez y época de desove de la reineta (Brama australis Valenciennes, 1836) en la costa central de Chile. Investigaciones Marinas 31(2): 17-24.

Mann G. 1954. Vida de los peces de aguas chilenas, 342 pp. Instituto de Investigaciones Veterinarias, Facultad de Filosofía y Educación, Universidad de Chile, Santiago.

Moreno CA. 1972. Nicho alimentario de la "vieja negra" (Graus nigra Philippi) (Osteichthyes, Labridae). Noticiario Mensual, Museo Nacional de Historia Natural 186: 5-6.

Moreno CA \& JC Castilla. 1980. Guía para el reconocimiento y observación de peces de Chile, 120 pp. Editora Nacional Gabriela Mistral, Valdivia. [Expedición a Chile]

Nikolsky GV. 1963. The ecology of fishes, 352 pp. Department of Ichthyology, Biology-Soil Faculty, Moscow State University, Moscow.

Oliva J, W Carbajal \& A Tresierra. 1986. Reproducción e histología de gónadas en peces, 151 pp. Departamento de Ciencias Biológicas, Sección de Recursos Acuáticos, Universidad Nacional de Trujillo, Trujillo.

Osorio A \& L Periche-Ruiz. 2001. Análisis del crecimiento de ovocitos de peces mediante el procesamiento digital de imágenes. Instituto Tecnológico de Chihuahua, ELECTRO 2001: 135-140.

Oyarzún C, J Chong \& M Malagueño. 1998. Fenología reproductiva en el jurel, Trachurus symmetricus (Ayres, 1855) (Perciformes, Carangidae) en el área de Talcahuano-Chile: 1982-1984. En: Arcos D (ed). Biología y ecología del jurel en aguas chilenas, pp. 67-75. Instituto de Investigaciones Pesqueras, Talcahuano.

Pardo A \& J Oliva. 1992. Estimación de la talla de primera madurez sexual de "caballa" (Scomber japonicus peruanus) en la zona norte de Chile durante el período de máxima actividad reproductiva. Investigación Pesquera, Chile 37: 97106. 
Pequeño G. 1989. Peces de Chile. Lista sistemática revisada y comentada. Revista de Biología Marina 24(2): 1-132.

Pequeño G. 2004. Peces litorales de las Islas Salas y Gómez, Chile, capturados durante el crucero CIMAR-ISLAS en 1999. Ciencia y Tecnología del Mar 27(1): 95-101.

Pequeño G \& F Olivera. 2005. Peces litorales de Chile, objeto de pesca: primer análisis de conjunto ¿hay en la pesquería litoral una amenaza a la diversidad ictiofaunística, que ha sido humanamente imperceptible e incalculable? En: Figueroa E (ed). Biodiversidad marina: Valoración, uso y perspectivas. ¿Hacia dónde va Chile?, pp. 507-538. Editorial Universitaria, Santiago.

Pequeño G \& S Sáez. 2000. Los peces litorales del archipiélago de Juan Fernández (Chile) endemismo y relaciones ictiogeográficas. Investigaciones Marinas 28: 27-37.

Pequeño G \& S Sáez. 2008. El estatus taxonómico de Doydixodon laevifrons (Tschudi, 1846) (Osteichthyes: Kyphosidae). Revista Peruana de Biología 15(1): 101-104.

Perez-Matus A \& A Buschmann. 2003. Sustentabilidad e incertidumbre de las principales pesquerías chilenas. Publicaciones Oceana 3: 1-163.

Ramos J. 1982. Contribución al estudio de la sexualidad del "lenguado", Solea solea (Linneo, 1758) (Pisces, Soleidae). Investigación Pesquera, España 46(2): 275-286.

Ramos J. 1983. Contribución al estudio de la oogénesis en el "lenguado", Solea solea (Linneo, 1758)(Pisces, Soleidae). Investigación Pesquera, España 47(2): 241-251.

Schneider F. 2008. Caracterización de la actividad pesquera de los buzos de la caleta de Pan de Azúcar, Región de Atacama, Chile. Tesis de Biología Marina, Facultad de Ciencias del Mar, Universidad Católica del Norte, Coquimbo, 52 pp.

SERNAPESCA 2010. Anuario estadístico de pesca, Servicio Nacional de Pesca, Ministerio de Economía, Fomento y Reconstrucción. Chile. [en línea] <http://www.sernapesca.cl>

Soto-Mamani R. 1985. Efecto del fenómeno de "El Niño"19821983 sobre ecosistemas de la I región. Investigación Pesquera, Chile 32: 199-206.
Sparre P \& SC Venema. 1995. Introducción a la evaluación de recursos pesqueros tropicales. Parte 1- Manual. FAO Documento Técnico de Pesca 306/1: 1-420.

Stepien CA. 1990. Population structure, diets and biogeographic relationships of a rocky intertidal fish assemblage in central Chile: high level of herbivory in a temperate system. Bulletin of Marine Science 47(3): 598-612.

Valdebenito I, S Peredo, K González \& C Sobarzo. 1995. Ciclo reproductivo anual del 'Huaiquil o Roncador' (Micropogonias furnieri Desmarest, 1823 Sin Micropogon manni Moreno, 1970) (Pisces: Sciaenidae) del lago Budi. Estudios Oceanológicos 14: 29-37.

Varas E \& FP Ojeda. 1990. Intertidial fish assemblages of the central Chilean coast: diversity, abundance and trophic patterns. Revista de Biología Marina 25(2): 59-70.

Vargas L \& G Pequeño. 2004. El estatus taxonómico de Graus fernandezianus Philippi 1887; Nuevo registro geográfico y comentarios sobre Graus nigra Philippi 1887 (Osteichthyes: Perciformes), en Chile. Gayana 68(1): 63-69.

Vargas M, RA Soto \& GL Guzmán. 1999. Cambios interanuales en la alimentación de peces submareales del norte de Chile entre los $20^{\circ} 11^{\prime}$ y $20^{\circ} 20^{\prime} \mathrm{S}$. Revista de Biología Marina y Oceanografía 34(2): 197-210.

Vial CI \& FP Ojeda. 1992. Comparative analysis of the head morphology of Pacific temperated Kyphosid fishes. Revista Chilena de Historia Natural 65(4): 471-483.

Vizziano D \& N Berois. 1990. Ciclo histológico del ovario de Macrodon ancylodon (Bloch \& Schneider, 1801) (Teleostei: Sciaenidae). Biología Pesquera 19: 39-47.

Zanuy S \& M Carrillo. 1973. Estudio histológico del ovario de la cabrilla (Paracentropiristis cabrilla L.) en relación con la ovogénesis. Investigación Pesquera, España 37(1): 117165.

Zárate P. 1997. Biología reproductiva del "pez espada" Xiphias gladius Linnaeus, 1758 en aguas chilenas. Tesis de Biología Marina, Facultad de Ciencias del Mar, Universidad Católica del Norte, $111 \mathrm{pp}$.

Recibido el 24 de marzo de 2009 y aceptado el 12 de mayo de 2010 